\section{Examination of MTM Opportunities in Pediatric Behavioral Health}

Medication therapy management (MTM) is a distinct group of services focused on optimizing medication-related therapeutic outcomes for individual patients; these services are independent of, but can occur with, the provision of a medication product. ${ }^{1}$ With the passage of the Medicare Prescription Drug, Improvement, and Modernization Act of 2003, which mandated providing MTM program (MTMP) services to a targeted group of Medicare Part D and Medicare Advantage beneficiaries, new opportunities arose for enhanced access to medication-related services. ${ }^{2,3}$ However, a majority of Americans are not covered by Medicare or do not meet the criteria for MTMP services, and thus may be unable to experience the health benefits of Medicarecovered MTMP services.

Outside of the Medicare statute, the term MTM is intended to cover a broad range of programs that address medication use in public and private sectors. ${ }^{4}$ Successful experiences with MTM over the past decade have shed light on ways in which to expand program scope. With health care policy and reform possibly moving towards universal coverage, MTM models can be designed based on past successes, as well as new opportunities identified beyond targeted Medicare beneficiaries. One such area of opportunity is pediatric behavioral health.

Common services offered by MTM programs include comprehensive medication therapy review, compilation of patient medication lists, individualized medication education mailings, telephonic or face-to-face pharmacotherapy consultations, designing medication action plans to address specific drug therapy needs with patients, and communicating with physician prescribers and other health providers., ${ }^{5,6}$ MTMP eligibility criteria specified by the Centers for Medicare and Medicaid Services (CMS) include multiple chronic conditions, multiple Part D medications, and anticipated Part D medication costs greater than a specified amount (\$4,000 per year in 2007).3,5 However, these services and eligibility criteria do not nearly cover the scope of need for patient access, service type, utilization of pharmacist expertise and provider recognition that could more fully optimize medicationrelated outcomes in a pediatric behavioral health population. We undertook an exploratory investigation, using a convenience sample, to assess how the lessons learned in MTM evaluations to date might be applied in a pediatric population receiving behavioral health services.

\section{Lessons Learned in MTM Program Experience}

Barnett et al. (2009) found that MTM services in community pharmacies evolved over 7 years (2000-2007) from patient education on acute medications to consultations with prescribers on chronic medication therapies. ${ }^{7}$ Some studies have shown positive benefit from MTM provided in specialty practice settings. ${ }^{8-10}$ Success has also been reported where MTM included pharmacist management of medication side effects and adverse drug reactions, and the tailoring of medication regimens to accommodate patient-specific needs. ${ }^{8}$ Meyer et al. observed that pharmacists can improve drug therapy by effectively identifying and addressing potential and actual medication-related problems (MRPs). ${ }^{11}$ MRP identification and the application of tested MTM strategies can potentially help maintain optimal drug therapy for any individual.

Cost minimization of prescribed medications is one goal of drug therapy optimization, but MTM encompasses a broad range of services and there are apparent opportunities for specialists including board-certified pharmacists for pharmacotherapy management. Most states permit some form of pharmacist-physician collaborative practice, ${ }^{12-14}$ but it seems that a greater proportion of MTM programs could still evolve to include collaborative practice or specific pharmacist prescribing arrangements to tailor medication regimens to patient-specific medication action plans.

\section{Assessment of the Potential for MTM in Pediatric Behavioral Health}

We performed a retrospective and exploratory chart review, based on a convenience sample, to assess the need for medication-related services in a primarily child and adolescent, nonMedicare population served by a private, ambulatory behavioral center providing primarily outpatient psychiatry, psychotherapy, and partial hospitalization services. We (a) collected demographic data; (b) defined eligibility for a potential MTM program for this patient population based on criteria that were derived from CMS, facility stakeholders, and pharmacist specialists; and (c) identified existing aspects of sound MTM that could potentially be applied to the sample. CMS criteria were adapted to define eligibility for MTM: (a) the presence of at least 1 psychiatric (Axis I) and 1 nonpsychiatric medical condition (Axis III) as the minimal criterion for the existence of multiple chronic conditions, and (b) multiple medications (4 or more, in this instance). Facility stakeholders had particular concern for monitoring medications consistent with Joint Commission medication management standards for behavioral health. From this position, additional criteria for patient selection were included: medications with potential for producing tardive dykinesia and high-dose psychopharmacology (above recommended dosage ranges). Pharmacist specialist criteria were based on MRPs as categorized by routine drug regimen review and services. Potential needs as documented in the records were considered as part of fulfillment of the 3 principles of sound MTM: (a) need for medication management assistance, (b) availability of health professionals who intervene with patients and physicians to improve drug regimens, and (c) established performance outcomes measures. ${ }^{5}$

From an average population of 600 patients served per month, a convenience sample of 60 (10\%) was identified and assessed as potential candidates over a 6 -week service period in February and March 2008. Data were collected from the patients' medical records, including age, gender, Axis I (psychiatric) and Axis III 
(nonpsychiatric, medical) diagnoses, primary care and pharmacy contact information, current medications, and potential MRPs. Six charts were not reviewed because the subjects were aged 18 years or older. Of 54 patients whose charts were reviewed in the final sample, 25 (46.3\%) were aged 7 to 12 years, 16 (29.6\%) were aged 13 to 17 years, and $13(24.1 \%)$ were less than 7 years of age (range 3-6 years). The majority $(70.4 \%, n=38)$ were male. The most common diagnoses in this population were (Axis I): attention deficit hyperactivity disorder (ADHD) and bipolar affective disorder (BPAD); and Axis III: asthma and/or allergies (38.9\%, $\mathrm{n}=21)$, history of hypertension and/or cardiac murmurs (22.2\%, $\mathrm{n}=12)$, and other conditions, primarily enuresis $(11.1 \%, \mathrm{n}=6)$. The number and proportion of subjects meeting MTM selection criteria were 14 (25.9\%) with at least 2 psychiatric diagnoses, 39 (72.2\%) with at least 1 psychiatric and 1 other medical diagnosis, and 37 (68.5\%) taking medications with tardive dykinesia potential or high-dose psychopharmacology.

Medication lists were present in all of the charts; however, an Axis III condition for example, might be listed in the multiaxial diagnosis, but the medications used to treat the condition were absent. Discrepancy between the medications patients are actually taking and those that are documented has been recognized as a problem to monitor in outpatient practices. ${ }^{15,16}$ The medical charts in the sample provided no evidence of a comprehensive medication therapy review including assessment of prescription and nonprescription medications, herbal products and dietary supplements, a service that would have been provided by a pharmacist developing a medication action plan in an MTM program. When queried, clinic staff indicated that they believed that medications would be screened for drug interactions at the local pharmacy where patients' prescriptions were filled and that they expected to be notified if there was a problem.

Medication selection, initiating, modifying, and administration were performed by physicians in this behavioral health services clinic, although clinical pharmacy specialists and advanced nurse practitioners were available on a consultant basis. Medication education, provided by a physician or case worker, was brief and limited usually to new prescriptions, with the expectation that patients would ask questions if they had any and obtain information at the time their prescription was filled. A history of medication noncompliance was documented in the medical record, and any efforts to promote medication adherence, if considered necessary, fell to a physician or case worker.

This exploratory chart review of a convenience sample of child and adolescent behavioral health patients identified more than one-quarter of patients with 2 or more psychiatric diagnoses and $72 \%$ with at least 1 Axis I psychiatric and 1 other Axis III medical diagnosis, suggesting opportunity to identify individuals with 2 or more chronic conditions who might be candidates for MTM intervention. More than one-half of the sample had incomplete medication regimen information. Maintaining such information is an MTM task that could yield benefit, particularly in coordination of care across multiple sites. The review also identified many medication-related tasks performed by different health professionals that might have been available to a greater number of patients, perhaps with fewer potential MRPs, had there been direct, routine pharmacist involvement at the facility in addition to or in place of the retail pharmacy. Some of these tasks could also potentially make use of specialist pharmacist training, for example, asthma disease management certification, or board certification as a psychiatric pharmacist.

Health care reform efforts should include expansion of MTM services. There is considerable opportunity for MTM program growth with existing eligible beneficiaries and in new populations not previously covered for these services. MTM growth should include expansion of Part D-covered MTMP and extension to Medicare Part B. Targets for MTM intervention should include pediatric behavioral health.

\section{Valerie Oji, PharmD, BCPP \\ Howard University College of Pharmacy, Nursing and Allied Health Sciences voji@howard.edu}

\section{DISCLOSURES}

The author reports no financial or other conflicts of interest associated with the subject of this letter. At the time that the exploratory assessment described in this letter was performed, she was Director, Center for Medication Use Outcomes \& Policy, Houston, Texas.

\section{REFERENCES}

1. American Pharmacists Association, National Association of Chain Drug Stores Foundation. Medication therapy management in community pharmacy practice: core elements of an MTM service (version 1.0). J Am Pharm Assoc (2003). 2005;45(5):573-79.

2. Public Law 108-173. The Medicare Prescription Drug, Improvement, and Modernization Act of 2003. December 8, 2003. Available at: http://www. treas.gov/offices/public-affairs/hsa/pdf/pl108-173.pdf. Accessed April 24, 2009.

3. American Society of Health System Pharmacists. A summary of medication therapy management programs in the Medicare Modernization Act and the Center for Medicare \& Medicaid Services. August 2006. Available at: http://www.ashp.org/s_ashp/docs/files/GAD_SummaryofMTMP0806.pdf. Accessed April 24, 2009.

4. Bluml BM. Definition of medication therapy management: development of profession-wide consensus. J Am Pharm Assoc (2003). 2005;45:566-72.

5. Academy of Managed Care Pharmacy. Sound medication therapy management programs, version 2.0 with validation study J Manag Care Pharm. 2008;14:(1 Suppl B):S2-S44. Available at: http://www.amcp.org/data/jmcp/ JMCPSuppB_Jan08.pdf.

6. McGivney MS, Meyer SM, Duncan-Hewitt W, Hall DL, Goode JV, Smith RB. Medication therapy management: its relationship to patient counseling, disease management and pharmaceutical care. J Am Pharm Assoc (2003). 2007;47(5):620-28. 
7. Barnett MJ, Frank J, Wehring H, et al. Analysis of pharmacist-provided medication therapy management (MTM) services in community pharmacies over 7 years. J Manag Care Pharm. 2009;15(1):18-30. Available at: http:// www.amcp.org/data/jmcp/018-031.pdf.

8. Hirsch JD, Rosenquist A, Best BM, Miller TA, Gilmer TP. Evaluation of the first year of a pilot program in community pharmacy: HIV/AIDS medication therapy management for Medi-Cal beneficiaries. J Manag Care Pharm. 2009;15(1):32-41. Available at: http://www.amcp.org/data/jmcp/032-041.pdf.

9. Cantwell-McNelis K, James CW. Role of clinical pharmacist in outpatient HIV clinics. Am J Health Syst Pharm. 2002;59:447-52.

10. March K, Mak M, Louie SG. Effects of pharmacists' interventions on patient outcomes in an HIV primary care clinic. Am J Health Syst Pharm. 2007;64(24):2574-78

11. Meyer B, Hill J, Trujillo G. Attaining pharmacist recognition in the United States. Am J Health Syst Pharm. 2008;65:2152-53.
12. Pharmacy Practice Research Roundtable. Advancing pharmacy practice through research: a 2004 perspective. J Am Pharm Assoc. 2004;44(5). Available at: http://www.medscape.com/viewarticle/491040. Accessed May 16, 2009.

13. Punekar, Y, Lin W. Thomas J III. Progress of collaborative practice: status of state laws and regulations and perceived impact of collaborative practice. J Am Pharm Assoc. 2003;43:503-10.

14. Alliance for Pharmaceutical Care. Pharmacists for quality patient care. Washington D.C.: Alliance for Pharmaceutical Care; 2003.

15. Elder NC. Patient safety in the physician office setting. Agency for Healthcare Research and Quality. Available at: http://www.webmm.ahrq.gov/ perspective.aspx?perspectiveID=24. Accessed May 16, 2009.

16. Bedell SE, Jabbour S, Goldberg R, et al. Discrepancies in the use of medications: their extent and predictors in an outpatient practice. Arch Intern Med. 2000;160:2129-34. Available at: http://archinte.ama-assn.org/cgi/ reprint/160/14/2129. Accessed May 20, 2009. 